\title{
Análisis de capas PML en una región de campo electromagnético utilizando el Método de Diferencias Finitas en el Dominio del Tiempo
}

\section{Analysis of PML layers in an electromagnetic field region using the Finite Differences in the Time Domain Method}

\author{
CRUZ-ORDUÑA, Maria Ines $\dagger^{*}$, MORALES-MENDOZA，Luis Javier, CORTEZ-DOMINGUEZ, \\ Cristobal y LAGUNA-CAMACHO, Juan Rodrigo
}

Universidad Veracruzana, Facultad de Ingeniería Mecánica y Eléctrica, Región Poza Rica - Tuxpan

ID $1^{\mathrm{er}}$ Autor: Maria Ines, Cruz-Orduña / ORC ID: 0000-0002-8771-0771, CVU CONACYT ID: 347038

ID $1^{\text {er }}$ Coautor: Luis Javier, Morales-Mendoza / ORC ID: 0000-0002-8580-2752, CVU CONACYT ID: 39020

ID $2^{\circ}$ Coautor: Cristóbal, Cortez-Domínguez / ORC ID: 0000-0002-5720-4405, CVU CONACYT ID: 972957

ID 3er Coautor: Juan Rodrigo, Laguna-Camacho / ORC ID: 0000-0003-0974-5204, CVU CONACYT ID: 100845

DOI: $10.35429 / \mathrm{JTO} .2019 .12 .3 .20 .26$

Recibido 04 de Octubre, 2019, Aceptado, 27 de Noviembre, 2019

\begin{abstract}
Resumen
El método de Diferencias Finitas en el Dominio del tiempo (FDTD) actualmente es uno de los métodos de electromagnetismo más utilizados en la simulación de campo electromagnético, sin embargo, para poder obtener resultados confiables de esta simulación es necesario contar con las condiciones de frontera óptimos. Las Capas Perfectamente Acopladas (PML) se caracteriza por la descomposición del campo transversal a la dirección de propagación en sus proyecciones rectangulares, y el empleo de conductividad tanto eléctrica como magnética en las capas numéricas para la absorción de los campos electromagnéticos. Las capas PML se caracterizan mediante valores de permitividad, permeabilidad y conductividad que se asignan a cada una de las ecuaciones según su posición en la región. Debido a que se trata del mismo conjunto de ecuaciones no hay necesidad de utilizar condiciones de conexión en la unión de la región de análisis con la región de absorción PML, lo que proporciona una transición natural en toda la región. En el trabajo se presenta el estudio de la diferencia entre la región de cálculo con capas PML y sin ellas.
\end{abstract}

FDTD, PML, Electromagnetismo

\begin{abstract}
The Finite Difference in Time Domain (FDTD) method is currently one of the most used electromagnetism methods in electromagnetic field simulation, however, in order to obtain reliable results from this simulation it is necessary to have optimal boundary conditions. Perfectly Coupled Layers (PML) is characterized by the decomposition of the transversal field to the direction of propagation in its rectangular projections, and the use of both electrical and magnetic conductivity in the numerical layers for the absorption of electromagnetic fields. The PML layers are characterized by values of permittivity, permeability and conductivity that are assigned to each of the equations according to their position in the region. Because it is the same set of equations there is no need to use connection conditions at the junction of the analysis region with the PML absorption region, which provides a natural transition throughout the region. This paper presents the study of the difference between the calculation region with and without PML layers.
\end{abstract}

FDTD, PML, Electromagnetism

Citación: CRUZ-ORDUÑA, Maria Ines, MORALES-MENDOZA, Luis Javier, CORTEZ-DOMINGUEZ, Cristobal y LAGUNA-CAMACHO, Juan Rodrigo. Análisis de capas PML en una región de campo electromagnético utilizando el Método de Diferencias Finitas en el Dominio del Tiempo. Revista de Operaciones Tecnológicas. 2019 3-12: 20-26

\footnotetext{
* Correspondencia del Autor (Correo electrónico: inesorduna@ gmail.com)

$\dagger$ Investigador contribuyendo como primer autor.
} 


\section{Introducción}

En los últimos años, se ha desarrollado la implementación del método de diferencias finitas en el dominio del tiempo para distintos modelados electromagnéticos, el método de diferencias finitas centrales en el dominio del tiempo es particularmente útil para problemas de propagación de ondas en dominios rectangulares. Su conveniencia sobre otras definiciones tiene que ver con su implementación computacional y sus expresiones matemáticas. Este trabajo es indispensable para poder realizar los análisis de forma correcta y sin reflexiones dentro de la región de cálculo, debido a que estas reflexiones generan interferencia con la propagación que se está generando continuamente.

\section{Método de diferencias finitas en el dominio del tiempo}

El método de Diferencias Finitas en el Dominio del Tiempo se basa en los principios del cálculo, donde existe una función $\mathrm{f}(\mathrm{x})$ continua en un intervalo finito, el cual puede ser particionado en una sucesión de puntos discretos, su derivada puede ser aproximada numéricamente mediante el promedio de su variación ante un pequeño incremento dentro de un intervalo de longitud $2 \Delta x$. (ecuación 1).

La figura 1 muestra la forma gráfica de la ecuación 1, donde la curva en color azul representa la función origina, la línea punteada representa la derivada exacta y la línea color rojo representa la aproximación de la derivada mediante diferencias centrales. [Cruz, 2018]

$f(x) \approx \frac{f(x+\Delta x)-f(x-\Delta x)}{2 \Delta x}$

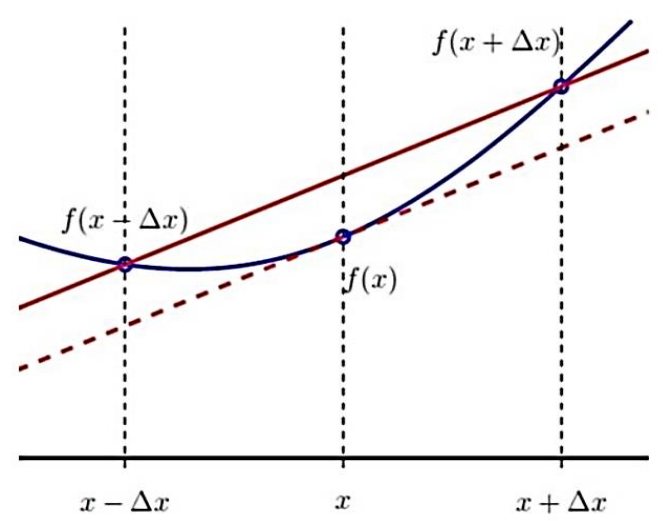

Figura 1 Aproximación de la derivada mediante diferencia central

Fuente: María Inés Cruz Orduña, 2018

ISSN 2523-6806

ECORFAN® Todos los derechos reservados
En las siguientes figuras se muestra en forma de ejemplos las gráficas de las aproximaciones con una función trigonométrica (figura 2) y función exponencial (figura 3), así como el código de Matlab que se empleó para obtenerlo.

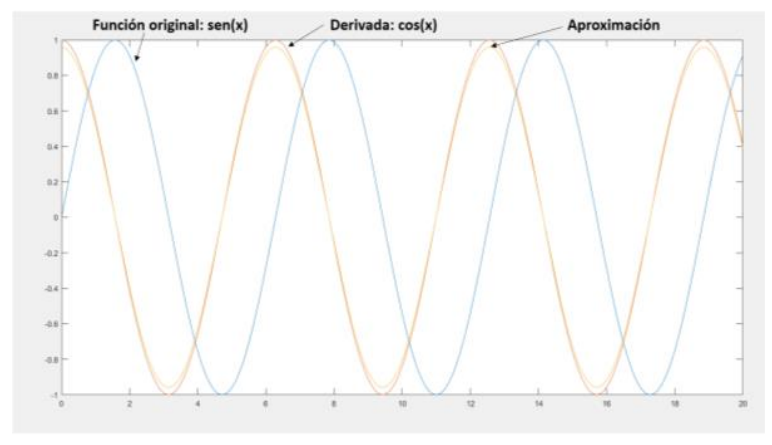

Figura 2 Ejemplo de la aproximación central con la función seno

Fuente: Elaboración Propia

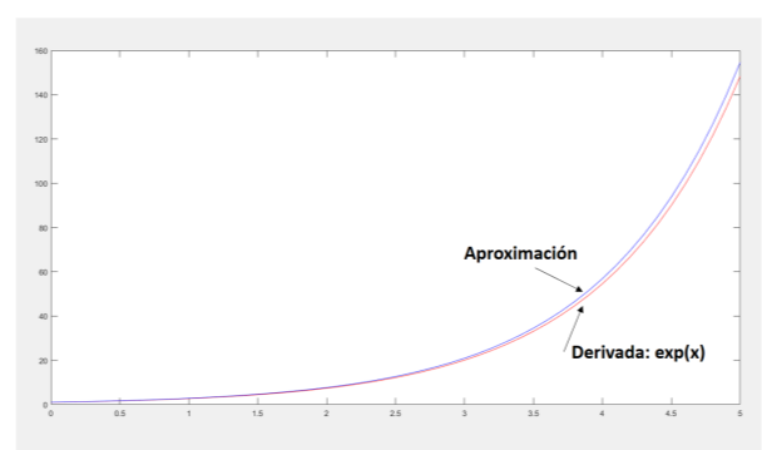

Figura 3 Ejemplo de la aproximación central con la función exponencial

Para poder implementar el algoritmo se debe describir la ecuación de onda, la cual se pude definir con la ecuación 2 , donde $u(x, t)$ es la función con dependencia de espacio y tiempo y c la velocidad de la luz.

$\nabla^{2} u(x, t)=c^{2} \frac{\partial^{2} u(x, t)}{\partial t^{2}}$

Para definir numéricamente la función solución se realizan dos suposiciones, una es que la función existe y la segunda es que tiene $n$ ésima derivada continua en un intervalo finito $I=\left(x_{i}-\Delta x_{i}, x_{i}+\Delta x_{i}\right)$. Para desarrollar esta ecuación, utilizamos la expansión de Taylor (ecuación 3) en ambos lados a un tiempo constante $\mathrm{t}_{\mathrm{n}}$

$u\left(x_{i}+\Delta x_{i}, t_{n}\right)$

$=u\left(x_{i}, t_{n}\right)+\Delta x \frac{\partial u}{\partial x}\left(x_{i}, t_{n}\right)+\frac{(\Delta x)^{2} \partial^{2} u}{2 \partial x^{2}}\left(x_{i}, t_{n}\right)$

$+\frac{(\Delta x)^{3} \partial^{3} u}{6 \partial x^{3}}\left(x_{i}, t_{n}\right)+\frac{(\Delta x)^{4} \partial^{4} u}{24 \partial x^{4}}\left(x_{i}, t_{n}\right)$

$+O\left(\Delta x^{5}\right)$

CRUZ-ORDUÑA, Maria Ines, MORALES-MENDOZA, Luis Javier, CORTEZ-DOMINGUEZ, Cristobal y LAGUNA-CAMACHO, Juan Rodrigo. Análisis de capas PML en una región de campo electromagnético utilizando el Método de Diferencias Finitas en el Dominio del Tiempo. Revista de Operaciones Tecnológicas. 2019 
Después de ello, es necesario sumar y ordenar las expresiones para despejar el término espacial de segundo orden, tal como se muestra en la ecuación 4. [Benavides, 2012]

$$
\frac{\partial^{2} u\left(x_{i}, t_{n}\right)}{\partial x^{2}}=\frac{u_{i+1}^{n}-2 u_{i}^{n}+u_{i-1}^{n}}{\Delta x^{2}}+O\left(\Delta x^{3}\right)
$$

La notación de subíndices y superíndices utilizados en la ecuación anterior, es la forma tradicional de escribir una ecuación en Diferencias Finitas, sabiendo que $u_{i}^{n}=$ $u\left(x_{i}, t_{n}\right)$, donde se define el valor de la función $\mathrm{u}$ en el punto discreto $\mathrm{Xi}$ al instante tn. Si se desarrolla la expansión temporal para la variable $t$ igual que el realizado en las derivadas espaciales se obtiene la siguiente ecuación.

$$
\frac{\partial^{2} u\left(x_{i}, t_{n}\right)}{\partial t^{2}}=\frac{u_{i}^{n+1}-2 u_{i}^{n}+u_{i}^{n-1}}{\Delta t^{2}}+O\left(\Delta t^{2}\right)
$$

\section{Discretización de las Ecuaciones de Maxwell con modo de propagación transversal magnético}

Las Ecuaciones de Maxwell representan las leyes de la Electricidad y el Magnetismo, éstas pueden predecir la existencia de ondas electromagnéticas (patrones de recorrido de campos eléctricos y magnéticos), las cuales viajan con una rapidez $c=\frac{1}{\sqrt{\mu_{0} \varepsilon_{0}}}=3 \times 10^{8} \mathrm{~m} /$ $s$, la rapidez de la luz. Además, la teoría muestra que dichas ondas son radiadas por cargas aceleradas.

Por simplicidad se presentan las ecuaciones de Maxwell como se aplican en el espacio libre, es decir, en ausencia de cualquier material dieléctrico o magnético. Las cuatro Ecuaciones son las mencionadas de la ecuación 6 a 9.

$\oint_{S} E \cdot d A=\frac{Q}{\epsilon_{0}}$

$\oint_{S} B \cdot d A=0$

$\oint E \cdot d s=-\frac{d \Phi_{B}}{d t}$

$\oint B \cdot d s=\mu_{0} I+\epsilon_{0} \mu_{0} \frac{d \Phi_{E}}{d t}$
Donde la ecuación 6 se refiere a la Ley de Gauss para campo Eléctrico, y la cuál describe que, el flujo eléctrico total a través de cualquier superficie cerrada es igual a la carga neta dentro de esa superficie dividida por $\epsilon_{0}$; la ecuación 7 es la Ley de Gauss para campo Magnético y establece que el flujo magnético neto a través de una superficie cerrada es cero; la ecuación 8 es la Ley de Faraday, ésta describe la creación de un campo eléctrico por un flujo magnético variable y por último, la ecuación 9 , es la Ley de Ampere (también conocida como Ley de Ampere-Maxwell) la cuál describe la creación de campo magnético a partir de campos y corrientes eléctricas.

Sin embargo, estas mismas ecuaciones, se pueden transformar para el caso en el que se analiza una región de cálculo sin fuentes de corriente eléctrica ni magnética, pero que puedan hallarse materiales que absorban energía de campos eléctricos y magnéticos. Las ecuaciones, por lo tanto, se convierten a la forma de las ecuaciones 10 a 13 .

$$
\begin{aligned}
& \nabla \cdot \overrightarrow{\boldsymbol{D}}=0 \\
& \nabla \cdot \overrightarrow{\boldsymbol{B}}=0 \\
& \frac{\partial}{\partial t} \overrightarrow{\boldsymbol{B}}=-\nabla \times \overrightarrow{\boldsymbol{E}}-\overrightarrow{\boldsymbol{M}} \\
& \frac{\partial}{\partial t} \overrightarrow{\boldsymbol{D}}=\nabla \times \overrightarrow{\boldsymbol{H}}-\overrightarrow{\boldsymbol{J}}
\end{aligned}
$$

Donde, $\overrightarrow{\boldsymbol{E}}$ representa la intensidad de campo eléctrico (volts/metro), $\overrightarrow{\boldsymbol{D}}$ a la densidad de flujo eléctrico (coulomb/metro $\left.{ }^{2}\right), \overrightarrow{\boldsymbol{H}}$ la intensidad de campo magnético (amperes/metro), $\overrightarrow{\boldsymbol{B}}$ la densidad de flujo magnético (webers/metro ${ }^{2}$ ), $\overrightarrow{\boldsymbol{J}}$ la densidad de corriente eléctrica (amperes/metro), $\overrightarrow{\boldsymbol{M}}$ es la densidad de corriente magnética equivalente (volts/metro).

Una vez establecidas las consideraciones anteriores, se obtienen las ecuaciones rotacionales de Maxwell para medios lineales, isotrópicos y no dispersivos con pérdidas. La descomposición de estas ecuaciones en sus componentes vectoriales, en este caso cartesianos, forman la base para la construcción del algoritmo FDTD y de las condiciones de absorción de frontera PML que se muestran en las ecuaciones 14 y 15. [Calderón, 2015] 
$\frac{\partial}{\partial t} \overrightarrow{\boldsymbol{H}}=-\frac{1}{\mu} \nabla \times \overrightarrow{\boldsymbol{E}}-\frac{1}{\mu}\left(\overrightarrow{\boldsymbol{M}}_{\text {fuente }}+\sigma^{*} \overrightarrow{\boldsymbol{H}}\right)$

$\frac{\partial}{\partial t} \overrightarrow{\boldsymbol{E}}=\frac{1}{\varepsilon} \nabla \times \overrightarrow{\boldsymbol{H}}-\frac{1}{\varepsilon}\left(\overrightarrow{\boldsymbol{J}}_{\text {fuente }}+\sigma \overrightarrow{\boldsymbol{E}}\right)$

Se puede notar que para el algoritmo FDTD no se muestran la Ley de Gauss para campo eléctrico ni para campo magnético; esto se debe a que estas relaciones son una consecuencia teórica directa de las ecuaciones rotacionales. Para este propósito las celdas espaciales FDTD deben estar estructuradas de tal manera que las relaciones de la Ley de Gauss se encuentren implícitas en las posiciones de los componentes vectoriales $\overrightarrow{\boldsymbol{E}}$ y $\overrightarrow{\boldsymbol{H}}$ y en las derivadas espaciales numéricas sobre estos componentes que modelan la acción del operador rotacional.

Para realizar el modelado de la región del algoritmo, se emplea la celda de Yee, en esta celda las componentes $\overrightarrow{\boldsymbol{E}}$ y $\overrightarrow{\boldsymbol{H}}$ están centrados en el espacio de tal manera que cada componente $\overrightarrow{\boldsymbol{H}}$ está rodeado por cuatro componentes de $\overrightarrow{\boldsymbol{E}}$ circulantes, tal como lo muestra la figura 4 .
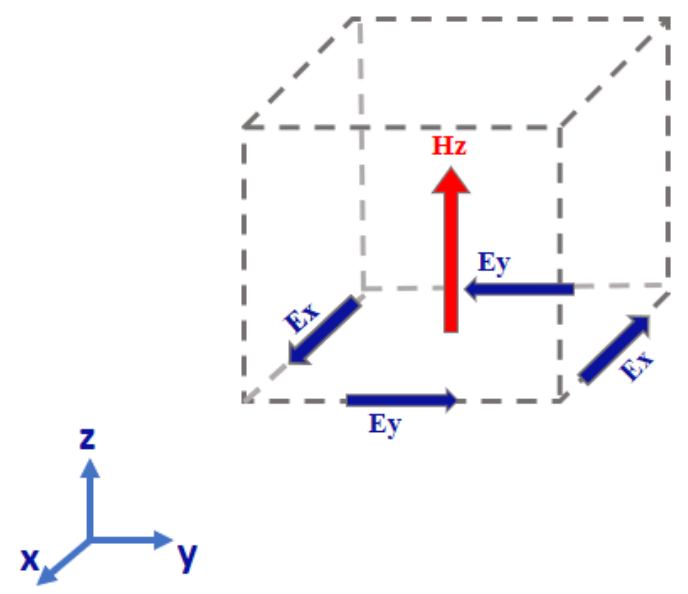

Figura 4 Celda de Yee

Fuente: Elaboración Propia

De forma continua, la celda de Yee se puede replicar varias veces dentro de la región de cálculo para formar un mallado en el cual se pueda analizar el comportamiento electromagnético en cada una de las celdas o de forma conjunta. En la figura 5 se muestra un ejemplo del mallado de la región de cálculo.
Diciembre, 2019 Vol.3 No.12 20-26

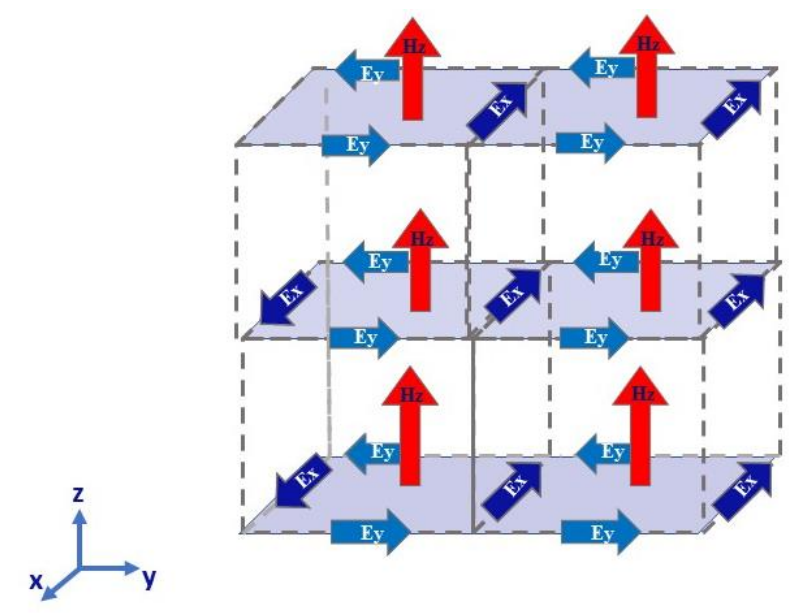

Figura 5 Mallado de las celdas de Yee para formar la región de cálculo

Fuente: Elaboración Propia

Aplicando los conceptos vistos en el apartado introductorio del método de diferencias finitas en el dominio del tiempo se puede obtener una formulación en diferencia finita central de las ecuaciones rotacionales de Maxwell (ecuaciones 14 y 15).

Apoyándose en la figura 5, se realiza la sustitución de las ecuaciones diferenciales centrales por las derivadas espacio-temporal evaluadas en el punto central de la tabla 1.

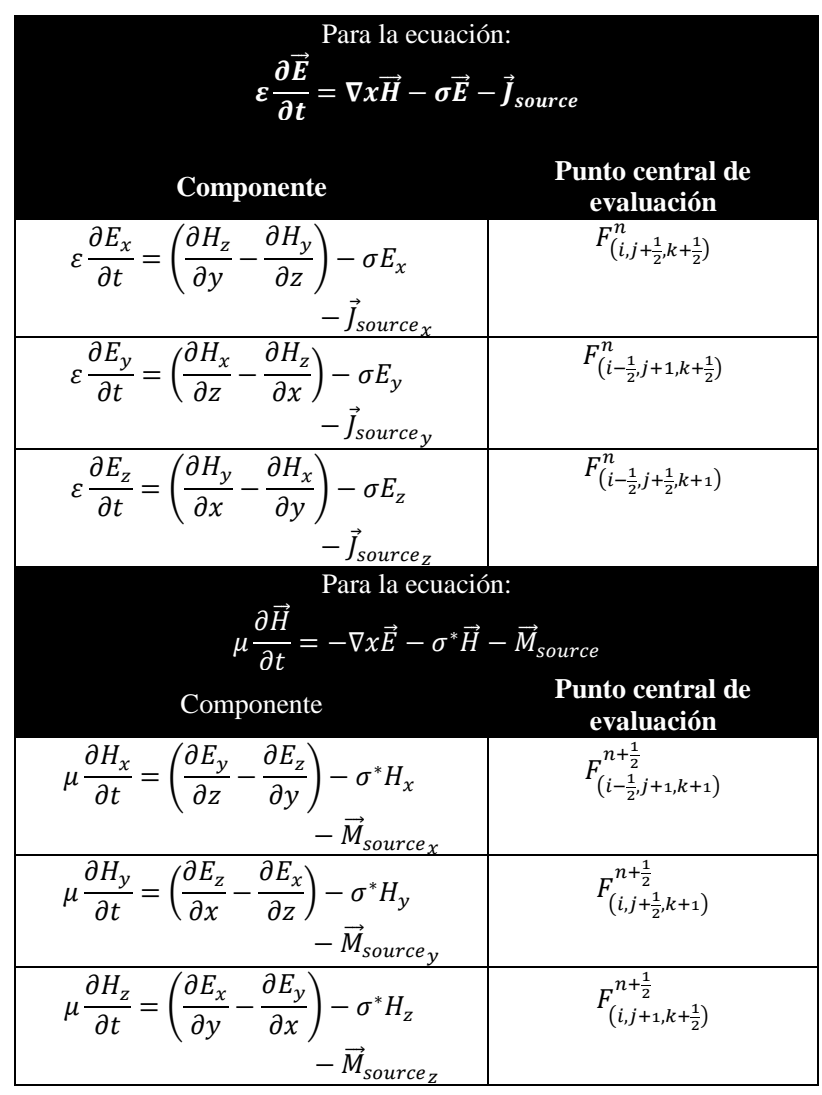

Tabla 1 Punto central de evaluación en diferencia finita central

Fuente: Elaboración Propia 
A continuación, en la tabla 2 se muestra la gráfica de la posición de cada punto central para las componentes de la tabla 1, cabe mencionar que esta representación gráfica es simplemente para ubicar el contexto espacial de los puntos centrales en el espacio.
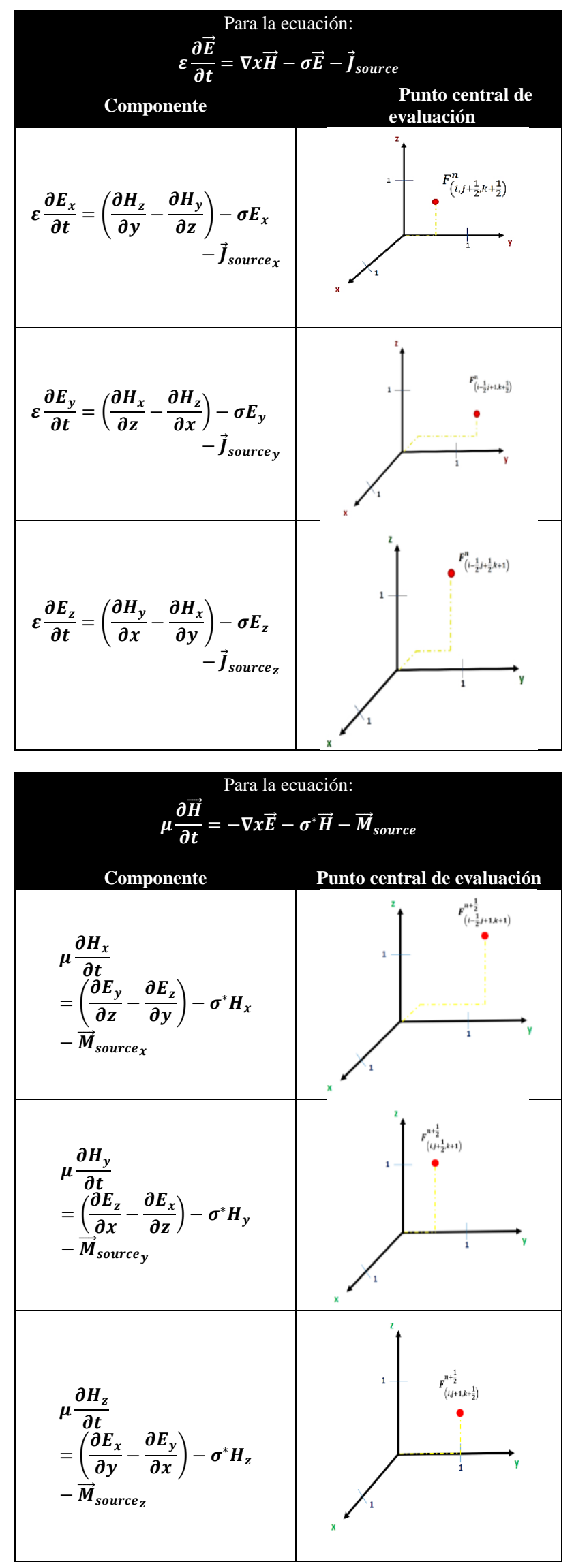

Tabla 2 Gráfica del punto central de las ecuaciones
De la misma forma, para la representación temporal se aplica el mismo método con el punto central temporal indicado en la tabla 1. Esto se representa a continuación en las figuras 6 y 7 .

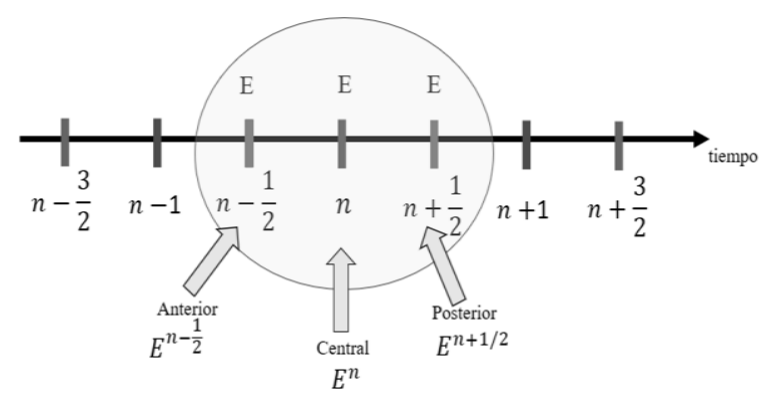

Figura 6 Referencia de la diferencia central temporal para el campo eléctrico

Fuente: Elaboración Propia

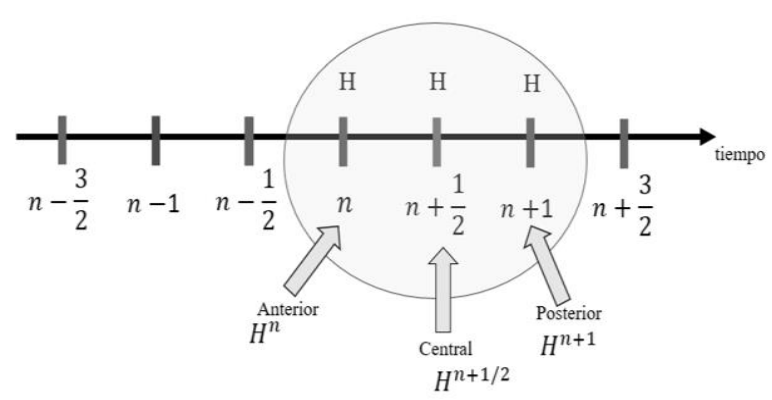

Figura 7 Referencia de la diferencia central temporal para el campo magnético

Fuente: Elaboración Propia

A partir de estas aproximaciones, se puede encontrar un sistema de ecuaciones en diferencia finita central, donde un nuevo valor de campo electromagnético en cualquier punto de la malla depende únicamente de su valor previo, los valores previos de las componentes de campo en los puntos adyacentes y de las fuentes y consumos de corriente eléctrica y magnética conocidas.

A continuación, en la ecuación 16 se muestra a manera de ejemplo, la ecuación por diferencias finitas de la componente Ex.

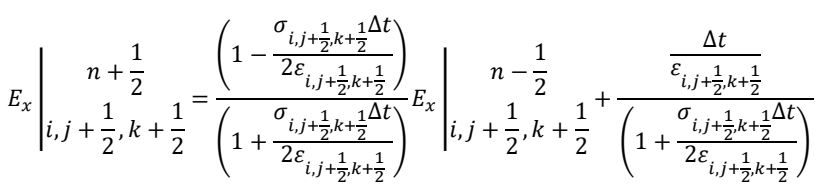

$\left(\frac{\left.H_{z}\left|\begin{array}{c}n \\ n\end{array}\right| \begin{array}{c}n, j+1, k+\frac{1}{2}-H_{z} \\ \Delta y\end{array}\right|_{i, j, k+\frac{1}{2}} ^{n}}{\Delta y}-\frac{\left.H_{y}\right|_{i, j+\frac{1}{2}, k+1} ^{n}-\left.H_{z}\right|_{i, j+\frac{1}{2}, k} ^{n}}{\Delta z}\right.$ 


\section{Condición de frontera de absorción PML}

Actualmente se han desarrollado varias técnicas para implementar condiciones de frontera absorbentes en el método FDTD, la condición de frontera de absorción de Capas Perfectamente Acopladas (PML, por sus siglas en inglés) se caracteriza por la descomposición del campo transversal a la dirección de propagación en sus proyecciones rectangulares, y el empleo de conductividad tanto eléctrica como magnética en las capas numéricas para la absorción de los campos electromagnéticos. [Benavides, 2012]

En general las técnicas de condiciones de frontera absorbentes son empleadas según la naturaleza del problema y el nivel de precisión que se desea. El autor de la referencia 1 realiza un análisis comparativo de estas técnicas e incluso, propone un conjunto de ecuaciones reducidas para la condición de frontera de absorción PML. Comenta que las ecuaciones PML pueden reducirse a tres tipos de medios: conductor, espacio libre y absorbente ${ }^{2}$

Se propone utilizar ecuaciones divididas en la región FDTD para representar 3 medios distintos (C. Calderón, et. al):

- Un medio sin pérdidas especificando que $\sigma_{x}=\sigma_{y}=\sigma_{z}=0$ y $\sigma_{x}^{*}=\sigma_{y}^{*}=\sigma_{z}^{*}=0$,

Un medio eléctricamente conductivo si $\sigma_{x}=\sigma_{y}=\sigma_{z}=\sigma$ y $\sigma_{x}^{*}=\sigma_{y}^{*}=\sigma_{z}^{*}=0$, Un medio absorbente PML si se hace que $\sigma_{x}=\sigma_{y}=\sigma_{z}=\sigma \quad$ y $\sigma_{x}^{*}=\sigma_{y}^{*}=\sigma_{z}^{*}=$ $\sigma^{*}$.

El esquema de implementación de la condición de frontera de absorción de capa perfectamente acoplada propuesta por el autor se muestra en la figura 2.9.

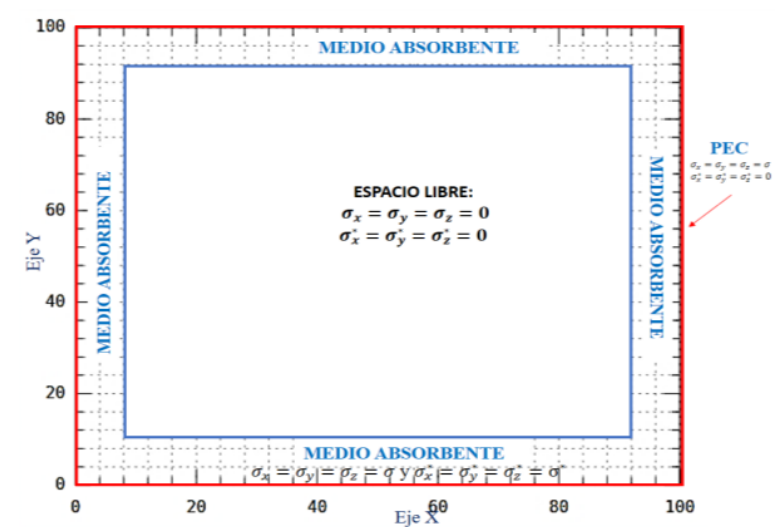

Figura 9 Esquema de implementación de la condición de frontera de absorción en una malla FDTD bidimensional Fuente: Elaboración Propia
Como resultado se obtiene el siguiente conjunto de cuatro ecuaciones (ecuaciones 17 a 20) que describen el comportamiento de los campos electromagnéticos. [Benavides, 2012]

$\mu \frac{\partial}{\partial t} H_{z y}+\sigma_{y}^{*} H_{z y}=\frac{\partial}{\partial y} E_{x}$

$\mu \frac{\partial}{\partial t} H_{z x}+\sigma_{x}^{*} H_{z x}=-\frac{\partial}{\partial x} E_{y}$

$\varepsilon \frac{\partial}{\partial t} E_{x}+\sigma_{y}^{*} E_{x}=\frac{\partial}{\partial y}\left(H_{z x}+H_{z y}\right)$

$\varepsilon \frac{\partial}{\partial t} E_{y}+\sigma_{x}^{*} E_{y}=-\frac{\partial}{\partial x}\left(H_{z x}+H_{z y}\right)$

Cabe hacer notar que el tipo de medio se asigna de manera predeterminada asignando valores de conductividad eléctrica y magnética equivalente, permitividad y permeabilidad a cada una de las ecuaciones según su posición en la región. Debido a que se trata del mismo conjunto de ecuaciones no hay necesidad de utilizar condiciones de conexión en la interfase de la región de análisis con la región de absorción PML, lo que proporciona una transición natural en la interfase.

\section{Resultados}

A continuación, en la tabla número 3 se muestra una comparación del comportamiento del campo electromagnético dentro de la región de cálculo sin las condiciones de frontera de absorción y con ella. Se observa en forma comparativa, las dos simulaciones comienzan perfectamente iguales, sin embargo, en la tercera gráfica de ambos casos se ve que llega a la periferia de la región de cálculo, casillas ocupadas en la región de cálculo para las capas de absorción perfectamente acopladas, recordando que son 24 filas en cada uno de los bordes de la región de cálculo.

En las gráficas de la cuarta posición (1350 iteraciones) se logra ver de forma clara y precisa el instante en el que llega la onda a la periferia de la región de cálculo, hasta esta iteración, las capas PML aún no han realizado la absorción de la onda incidente. A partir de ésta iteración, se muestra el efecto que tienen las capas PML dentro de la región de cálculo, pues mientras que en la gráfica sin PML ha reflejado por completo toda la onda electromagnética, generando interferencia en la región de cálculo en la región que tiene implementado las capas PML la ha absorbido prácticamente por completo.

CRUZ-ORDUÑA, Maria Ines, MORALES-MENDOZA, Luis Javier, CORTEZ-DOMINGUEZ, Cristobal y LAGUNA-CAMACHO, Juan Rodrigo. Análisis de capas PML en una región de campo electromagnético utilizando el Método de Diferencias Finitas en el Dominio del Tiempo. Revista de Operaciones Tecnológicas. 2019 
De tal manera que en la última comparación de las gráficas (2350 iteraciones) se logra observar el impacto que han tenido las capas PML en la región mostrada en la gráfica de la derecha, mientras que en la gráfica de la izquierda, la reflexión de la onda incidente generó interferencia con la onda reflejada generando así complejidad en la medición de los parámetros en la región de cálculo. [Cruz, 2018]

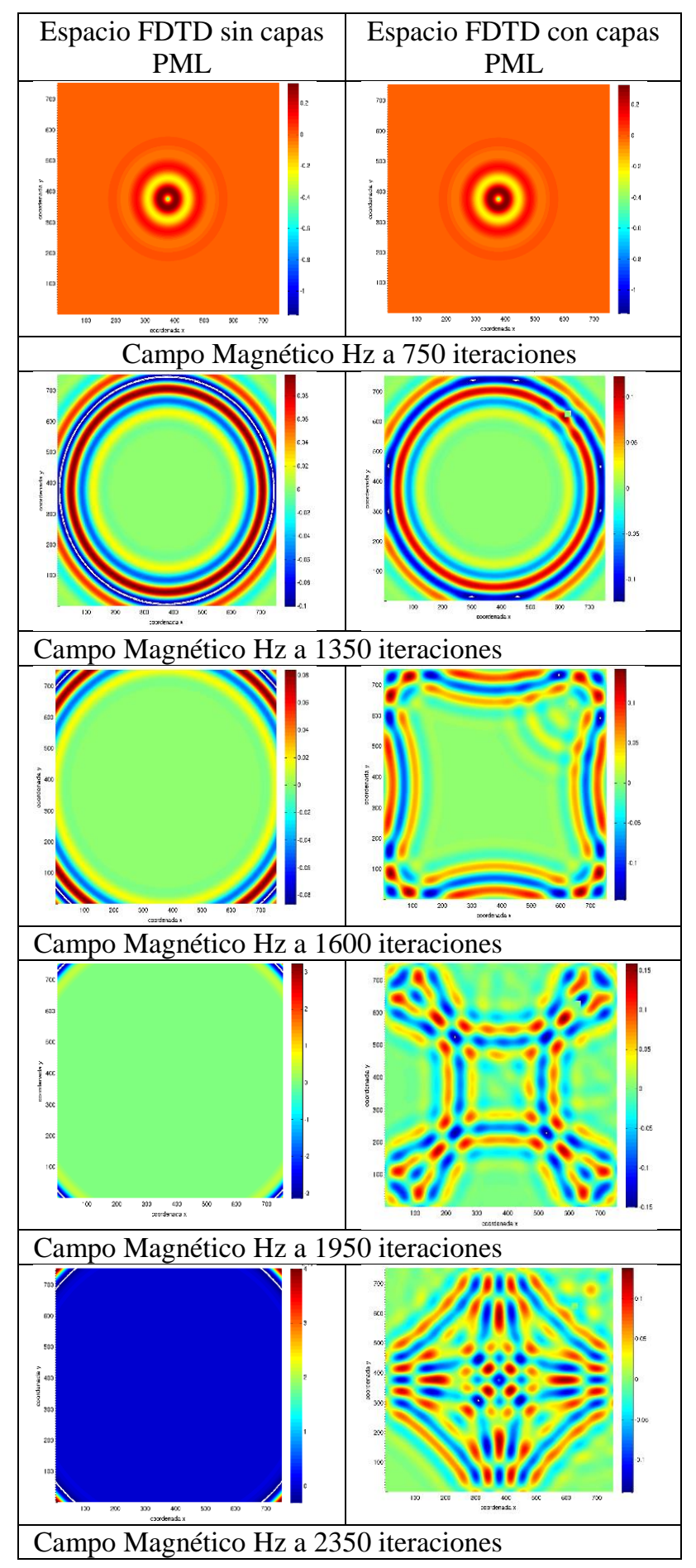

Tabla 3 Comparación de la implementación de capas PML, Fuente: Elaboración Propia

\section{Conclusiones}

Se logra diseñar e implementar el Método de Diferencias Finitas en el Dominio del Tiempo (FDTD) de forma correcta en la región de cálculo, empleando las técnicas para definir e implementar la región de capas perfectamente acopladas (PML). En las gráficas mostradas en el apartado de resultado, se logra apreciar la importancia de estas capas en la región de cálculo, pues sin estas regiones sería difícil definir con claridad los parámetros encontrados en la región debido a la interferencia que se genera entre la onda incidente y la onda reflejada por el perímetro de la región sin capas PML.

\section{Referencias}

Calderón Ramón, C. M. (2015). Análisis y modelado electromagnético de un arreglo circular de antenas con cuerpos opacos discretizados en su interior, utilizando el método de diferencias finitas en el dominio del tiempo. (1, Trad.) Ciudad de México: Tesis doctoral, Instituto Politécnico Nacional. Recuperado el 5 de Agosto de 2019

Cruz Orduña, M. I. (2018). Desarrollo de un sistema computacional para el modelado de tejido maligno utilizando el método de diferencias finitas en el dominio del tiempo (Vol. 1). Ciudad de México, México: Tesis doctoral del Instituto Politécnico Nacional. Recuperado el 5 de Agosto de 2019

Cruz, A. M. (2012). Análisis y modelado de la interacción de campo electromagnético con estructuras nanométricas utilizando técnicas de electromagnetismo computacional (Vol. 1). (A.

M. Benavides Cruz, Ed.) Ciudad de México, México: Tesis doctoral, Instituto Politécnico Nacional. Recuperado el 5 de Agosto de 2019 